\title{
Was kann ich da berechnen?
}

\section{Dr. med. Cl. K, KV Saarland:}

Wir haben seit einiger Zeit eine E-MailAdresse für die Praxis. Meine Diabetiker übermitteln ihre Blutzuckerwerte elektronisch und stellen auch ihre Fragen per E-Mail. In der Antwort berate ich zu den gestellten Fragen. Wie kann ich dies, wenn überhaupt berechnen?

Antwort: Im GKV-Bereich gibt es ja für die einzelne Beratung keine Vergütung mehr. In einzelnen KVen gibt es so genannte Kontaktziffern wie beispielsweise in Bayern die GOP 99 215. Hiermit soll die Anzahl der Kontakte erfasst werden. Die GOP ist jedoch vergütungsneutral.

Handelt es sich um einen Zweitkontakt bei einem chronisch Kranken - dazu zählen Diabetiker - kommt die GOP 03 212, Zuschlag zur Versichertenpauschale für die Behandlung eines Versicherten mit schwerwiegenden Erkrankung(en), in Frage. Diese GOP verlangt mindestens zwei ArztPatienten-Kontakte (APK), allerdings keinen persönlichen APK, sodass der Ansatz auch bei E-Mail-Kontakt möglich ist.

\section{Kann ich bei Privatpatienten mehr als den Regelsatz verlangen?}

Dr. med. Chr. H., Allgemeinärztin, Bayreuth: Insbesondere bei alten Patienten dauern meine Hausbesuche häufig 30 Minuten. Gibt es wenigstens bei Privatpatienten eine Möglichkeit, dies finanziell auszugleichen, beispielsweise mit Steigerung des Hausbesuches?

Antwort: Dies ist grundsätzlich möglich. Die Steigerung über das 2,3-Fache, was derzeit der Regelsatz ist, hinaus bedarf allerdings der gesonderten Begründung. Sie können im Einzelfall mit dem „erheblich über das übliche Maß hinausgehenden Zeitaufwand für Beratung oder Abklärung ..." begründen. Allerdings kann sicherlich nicht regelmäßig so begründet werden. Auch ein Privatpatient muss unter wirtschaftlichen Gesichtspunkten gesehen werden: Mit zwei auf den 3,5-fachen Satz gesteigerten Hausbesuchen kommt ohne weitere Leistungen lediglich ein Stundenumsatz von $€ 130,56$ zustande. Um einigermaßen angemessen wirtschaftlich zu arbeiten, müsste ein Umsatz von 250 $€$ pro Stunde angestrebt werden.

Bei psychisch alterierten Patienten kommen zusätzlich die GOP 804, 806, 849 in Frage, die selbstverständlich auch gesteigert werden
Im Bereich der GOÄ sieht es besser aus: Da auch per E-Mail eine „Interaktion“ zwischen Arzt und Patient zustande kommt, kann die GOP 1 abgerechnet werden. Wird die Anfrage im Auftrag des Arztes durch eine Mitarbeiterin erledigt, fällt lediglich die GOP 2 an. Wird die Anfrage durch den Arzt per Diktat erledigt, ist es wiederum die GOP 1, denn ein persönliches Eintippen der Antwort ist nicht die entscheidende Voraussetzung. können. Da Steigerungen bei der PKV sehr schnell zu einer intensiveren Prüfung der Gesamtliquidation führen und damit Rückfragen ins Haus stehen, sollten der Zeitaufwand und die Gründe für den zeitlichen Aufwand sorgfältig dokumentiert werden. Bei Untersuchungen, die den Inhalt der GOP 5 - im Hausbesuch enthalten - überschreiten, kann neben den GOP 6, 7, 8 der Zuschlag nach Buchstaben „A“ angesetzt werden. 